Author's Accepted Manuscript of

Rossi, A.; Lanzetta, M.: Roundness: a closed form upper bound for the centroid to minimum zone center distance by worst-case analysis, Measurement, vol. 46, n. 7, August 2013, pp. 2251-2258 (8), ISSN:0263-2241,

DOI:10.1016/j.measurement.2013.03.025. http://www.sciencedirect.com/science/article/pii/S0263224113000924

Submitted May 2012, revisions completed 11 December 2012, available online 6 April 2013

\title{
Roundness: a closed form upper bound for the centroid to minimum zone center distance by worst-case analysis
}

\section{Andrea Rossi and Michele Lanzetta}

Department of Civil and Industrial Engineering

University of Pisa, Largo Lazzarino, 56122 Pisa, Italy

Email: lanzetta@unipi.it tel.: +390502218122 fax: +390502218065

\begin{abstract}
The minimum zone tolerance (MZT) meets the ISO 1101 definition of roundness error: it determines two concentric circles that contain the roundness profile and such that the difference in radii is the least possible value.

This article provides theoretical evidence that the minimum size of the neighborhood of the centroid containing the minimum zone center is $\pi^{-1} E_{C}$, where $E_{C}$ is the roundness error related to the centroid, which can be evaluated in closed form.

The implications of such linear estimating are twofold: (i) locating the part center with a given tolerance, e.g. for manufacturing tasks, such as handling (peg-hole) or machining (centering) and (ii) providing a search area for minimum zone center-based algorithms, such as metaheuristics (GA, PSO etc.).
\end{abstract}

Keywords: minimum zone tolerance (MZT), centroid neighborhood, upper bound, MZC evaluation, circularity. 


\section{Introduction}

The evaluation of form errors of machined parts is fundamental in quality inspection to verify their conformance to the expected tolerances. The form tolerance is evaluated with reference to a Euclidean geometric feature, i.e. a circle in the case of roundness. Roundness is a typical geometric form to be inspected.

The most used criteria to establish the reference circle are: the least-squares method (LSQ), the maximum inscribed circle (MIC), the minimum circumscribed circle (MCC) and the minimum zone tolerance (MZT). The performance of minimum zone fitting algorithms has been reviewed in [1]. The use of a particular data fitting method depends on the required application; e.g., MIC and MCC can be used when mating is involved. The MZT meets the standard definition of roundness error, as reported in ISO 1101 [2] and therefore it prevents good parts to be rejected by data fitting and economic loss.

The MZT determines two concentric circles that contain the roundness profile and such that the difference in radii is the least possible value. This difference is the minimum zone roundness error $E_{M Z}$. Figure 1 shows two pairs of concentric circles that include the sample points centered respectively at $C_{1}$ and $C_{2}$ and where $\left(R_{1}-r_{1}\right)$ and $\left(R_{2}-r_{2}\right)$ are their difference in radii. If the minimum zone center is found, the minimum zone error can be determined as the corresponding roundness error. 


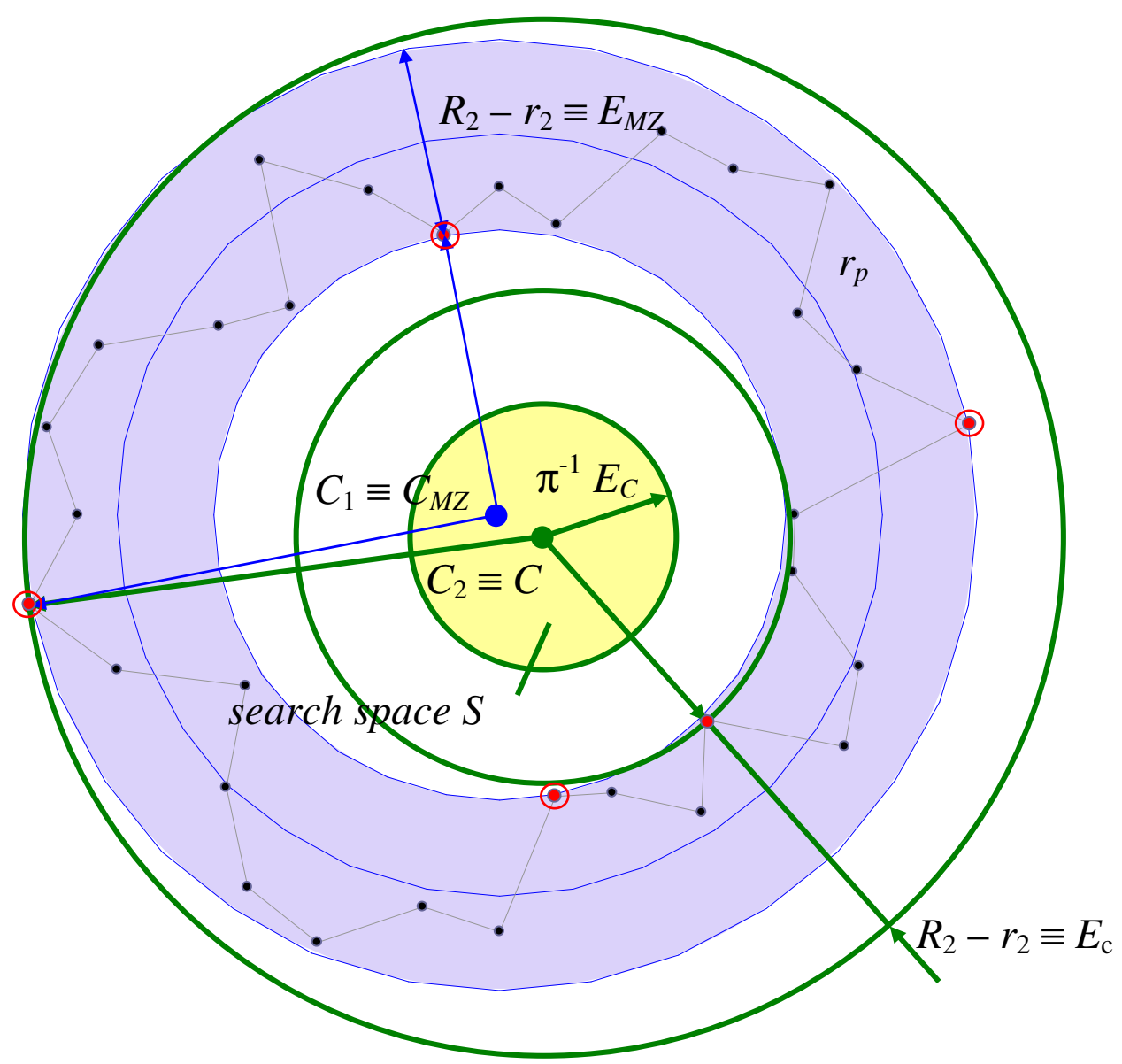

Figure 1: Minimum zone error $E_{M Z} . C_{1}$ and $C_{2}$ are possible locations of the centers of the two concentric circles. $\left(R_{1}-r_{1}\right)$ and $\left(R_{2}-r_{2}\right)$ are the differences in radii. $C_{1}$ becomes the minimum zone center $C_{M Z}$ if the minimal difference in radii $\left(R_{1}-r_{1}\right)$ coincides with $E_{M Z}$. Center-based algorithms (like metaheuristics) search the (unknown) neighborhood $S$ for $C_{M Z}$. The main purpose of this work is to provide center and radius of $S$, as indicated, where $C$ is centroid of the roundness profile $r_{p}$.

Errors are scaled for clarity.

About here

The MZT is the solution of the following optimization problem [3]:

$$
\min \left[\max _{\theta_{i}=i \times \frac{2 \pi}{n}, i=1, . ., n} r_{p}\left(x, y, \theta_{i}\right)-\min { }_{\theta_{i}=i \times \frac{2 \pi}{n}, i=1, . ., n} r_{p}\left(x, y, \theta_{i}\right)\right]
$$

subject to $(x, y) \in S$

where $S$ is the search space, $\theta_{i}=i \times \frac{2 \pi}{n}, i=1, \ldots, n$ are the angular locations of $n$ equiangular data of the roundness profile $r_{p}\left(x, y, \theta_{i}\right)$ of the reference circle of center $(x, y)$.

The solution of problem (1) is the minimum zone error defined as: 


$$
\begin{aligned}
E_{M Z} & =R_{\max }\left(C_{M Z}\right)-R_{\min }\left(C_{M Z}\right)= \\
& =\max _{\theta_{i}=i \times \frac{2 \pi}{n}, i=1, ., n} r_{p}\left(C_{M Z}, \theta_{i}\right)-\min _{\theta_{i}=i \times \frac{2 \pi}{n}, i=1, ., n} r_{p}\left(C_{M Z}, \theta_{i}\right)
\end{aligned}
$$

where $C_{M Z}=\left(x_{M Z}, y_{M Z}\right)$ is the minimum zone center.

The main purpose of this work is to provide a closed form upper bound of the distance between centroid $C_{n}$ and minimum zone center $C_{M Z}$. This evaluation can be used tout court as a first estimation of the minimum zone center position or as the starting point for a local search, e.g. as a search neighborhood of metaheuristics, such as genetic algorithms, particle swarm optimization etc. By reducing the search area the algorithm complexity and the computation time can be reduced. It seems that a theoretical analysis that provides a closed form expression for the roundness problem with the MZT criterion is only available by the authors [4], where the center of the search space $S$ is the centroid and the radius is the upper bound of the distance between centroid and minimum zone center; current lowest upper bound of about $0.43 E_{C_{n}}$ has been extrapolated asymptotically.

The search space definition represents the focus of current work. It will be shown that in the proposed approach it has been decreased by $27 \%$.

\section{Literature}

Two approaches to the MZT problem have been proposed in the literature: computational geometry techniques and solutions of a non linear optimization problem. The first approach is, in general, very computationally intensive, especially, when the number of data points is large. One of these methods is based on the Voronoi diagram [5] [6]. The second approach is based on the minimization of the $E_{M Z}$ as a function of $C_{M Z}$ (Figure 1). The inconvenience is that this function has several local minima consequently the exploration is computation intensive; for this reason it takes advantage of the search area reduction, which is the main purpose of current work. Some examples of center-based approaches are: the Chebyshev approximation [7], the simplex search / linear approximation [8] [9], the steepest descent algorithm [10], and metaheuristics like the particle swarm optimization (PSO) [11] [12], the simulated annealing (SA) [13], and genetic algorithms (GAs) [3] [14] [15].

Wang et al. [16] and Jywe et al. [17] presented a generalized non-linear optimization procedure based on the developed necessary and sufficient conditions to evaluate the roundness error. In order to meet the standards, the minimum zone reference circles should pass through at least four points of the roundness profile. This can occur in two cases: a) when three points lie on a circle and one point lies on the other circle (the 1-3 and the 3-1 criteria); b) when two points lie on each of the 
concentric circles (the 2-2 criterion, represented by the circled red points in Figure 1). The computation time required to meet these conditions increases exponentially with the dataset size. Gadelmawla [18] uses a heuristic approach to drastically reduce the number of sample points used by the min-max 1-3, 3-1 and 2-2 criteria.

Samuel and Shunmugam [19] established a minimum zone limaçon based on computational geometry to evaluate roundness error; with geometric methods, global optima are found by exhaustively checking every local minimum candidate. A mesh based method with starting center on the least square center, where the convergence depends on the number of mesh cross points, representing a compromise between accuracy and speed, was proposed by Xianqing et al. [20]. This and similar approaches require the approximate size of the search space.

The strategy to equiangular data on the roundness profile is generally adopted in the literature. Conversely, in a previous paper the authors developed a cross-validation method for small samples to assess the kind of manufacturing signature on the roundness profile in order to detect critical points such as peaks and valleys [21].

Only few contributions are currently available in the literature regarding the setting of the search space of the non linear optimization problem. The centroid is usually considered as the center of the search space. In [14] the search space is a square of fixed $0.2 \mathrm{~mm}$ side, in [3] it is $5 \%$ of the circle diameter [22], the side is determined by the distance of the farthest point and the nearest point from the mean center which is approximated to $2 E_{C_{n}}$ where $E_{C_{n}}$ is the roundness error related to the centroid of $n$ equiangular data. In [23] it is the rectangle circumscribed to the sample points.

\section{An upper bound for the centroid to minimum zone center distance}

In this section, we define a geometrical feature $F(\alpha)$ that represents a worst-case for the examined roundness problem. A formal theory is developed and presented below to find a closed form expression for $a$ theoretical (local) maximum (upper bound) of the distance between the centroid, $C=\left(x_{0}, y_{0}\right)$, and the minimum zone center, $C_{M Z}=\left(x_{M Z}, y_{M Z}\right)$.

The proposed theory is general, it does not take into account form deviations, e.g. it is not signature specific.

The proposed geometrical feature $F(\alpha)$ inspired from [4] is formed by two concentric-opposite arcs of circle shown in Figure 2 and described by: 


$$
F(\alpha)= \begin{cases}(x, 0), & \theta=0 \\ (R \cdot \cos \theta, R \cdot \sin \theta), & \theta \in\left(0, \frac{\pi}{2}+\alpha\right] \\ (r \cdot \cos \theta, r \cdot \sin \theta), & \theta \in\left(\frac{\pi}{2}+\alpha, \frac{3 \pi}{2}-\alpha\right) \\ (R \cdot \cos \theta, R \cdot \sin \theta), & \theta \in\left[\frac{3 \pi}{2}-\alpha, 0\right)\end{cases}
$$

where $0<r<R,-\frac{\pi}{2} \leq \alpha<\frac{\pi}{2}$ and $(x, 0)$ is a control point, which makes the feature open in $\theta=0$. By construction $\alpha=\theta+\frac{\pi}{2}$.

In Appendix, the effect of the variation of $x \in[0, R]$ on the minimum zone error is discussed.

It will be proven that for $x=r$ the control point forces the minimum zone center in $\left(x_{M Z}, y_{M Z}\right)=(0,0)$. This property is exploited by using the feature $F(\alpha)$ with the control point at $(r, 0)$ as displayed in Figure 2. 


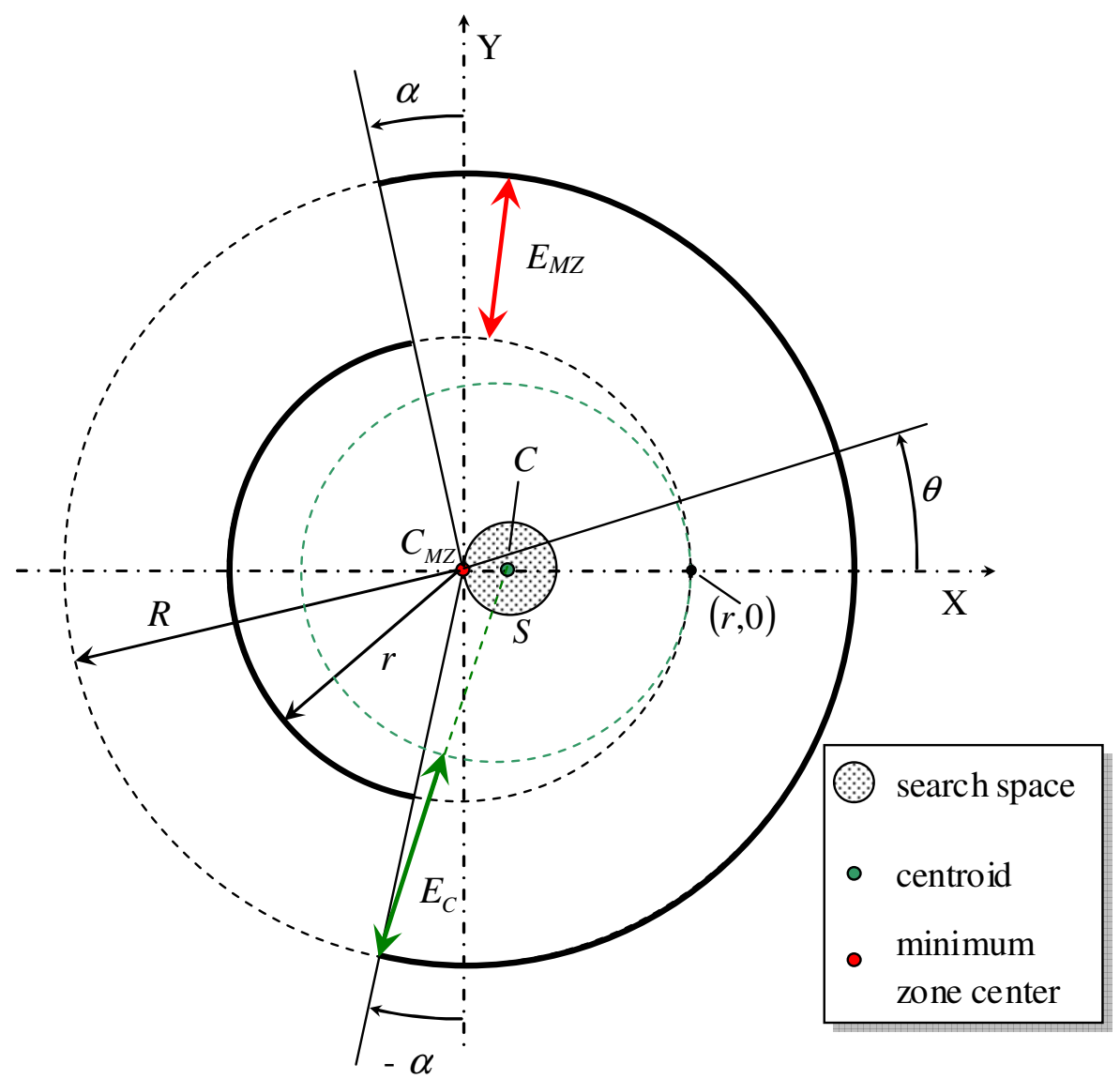

Figure 2: Worst-case for the distance (enhanced for clarity) between centroid $C$ and minimum zone center $C_{M Z}$, with the control point in $(r, 0)$

About here

It results that $F(0)$ is formed by two concentric-opposite semicircular arcs.

The feature $F\left(\frac{\pi}{2}\right)$ is a circumference of radius $R$; similarly, $F\left(-\frac{\pi}{2}\right)$ is a circumference of radius $r$.

As the considered feature is symmetrical with respect to the $\mathrm{X}$ axis by a rotation of axes, without loss of generality in the remainder the $\mathrm{Y}$ component is neglected.

A number of properties of $F(\alpha)$ is given. They can be applied for each feature defined by expression (3) and rotated by $\frac{k}{2 \pi}, 0 \leq k<2 \pi$.

Lemma 1. Let $C$ be the centroid of $F(0)$, then $C=\left(\frac{R-r}{\pi}, 0\right)$ 
- The proof follows the fact that $F(0)$ is formed by two semicircular arcs of radii $R$ and $r$ and the centroids of these semicircular arcs are located on the $\mathrm{X}$ axis respectively at $C_{R}=\frac{2 \cdot R}{\pi}$ and $C_{r}=-\left(\frac{2 \cdot r}{\pi}\right)$. Obviously, the control point $(r, 0)$ has a negligible weight in the evaluation of $C \square$

Let $F_{R}(\alpha)$ and $F_{r}(\alpha)$ be the component features of radii $R$ and $r$, respectively, at the right and at the left side of $F(\alpha)$ as shown in Figure 3. Figure 3 (top) shows that $F(\alpha)$ is obtained by $F(0)$ removing (adding) a feature symmetric on the $\mathrm{X}^{-}$axis $\left(\mathrm{X}^{+}\right.$axis), if $\alpha>0(\alpha<0)$. The maximum asymmetry of $F(\alpha)$ about the $\mathrm{Y}$ axis is achieved by setting $\alpha=0$. In fact if $\alpha>0, F_{r}(\alpha)$ evaluated for $\frac{\pi}{2} \leq \theta \leq \frac{\pi}{2}+\alpha$ is mirrored to $F_{R}(\alpha)$ evaluated for $\frac{\pi}{2}-\alpha \leq \theta \leq \frac{\pi}{2}$ (Figure 3 bottom). Analogously if $\alpha<0 \quad F_{R}(\alpha)$ evaluated for $\alpha \leq \theta \leq \frac{\pi}{2}$ is mirrored to $F_{r}(\alpha)$ evaluated for $\frac{\pi}{2}+\alpha \leq \theta \leq \pi$ 


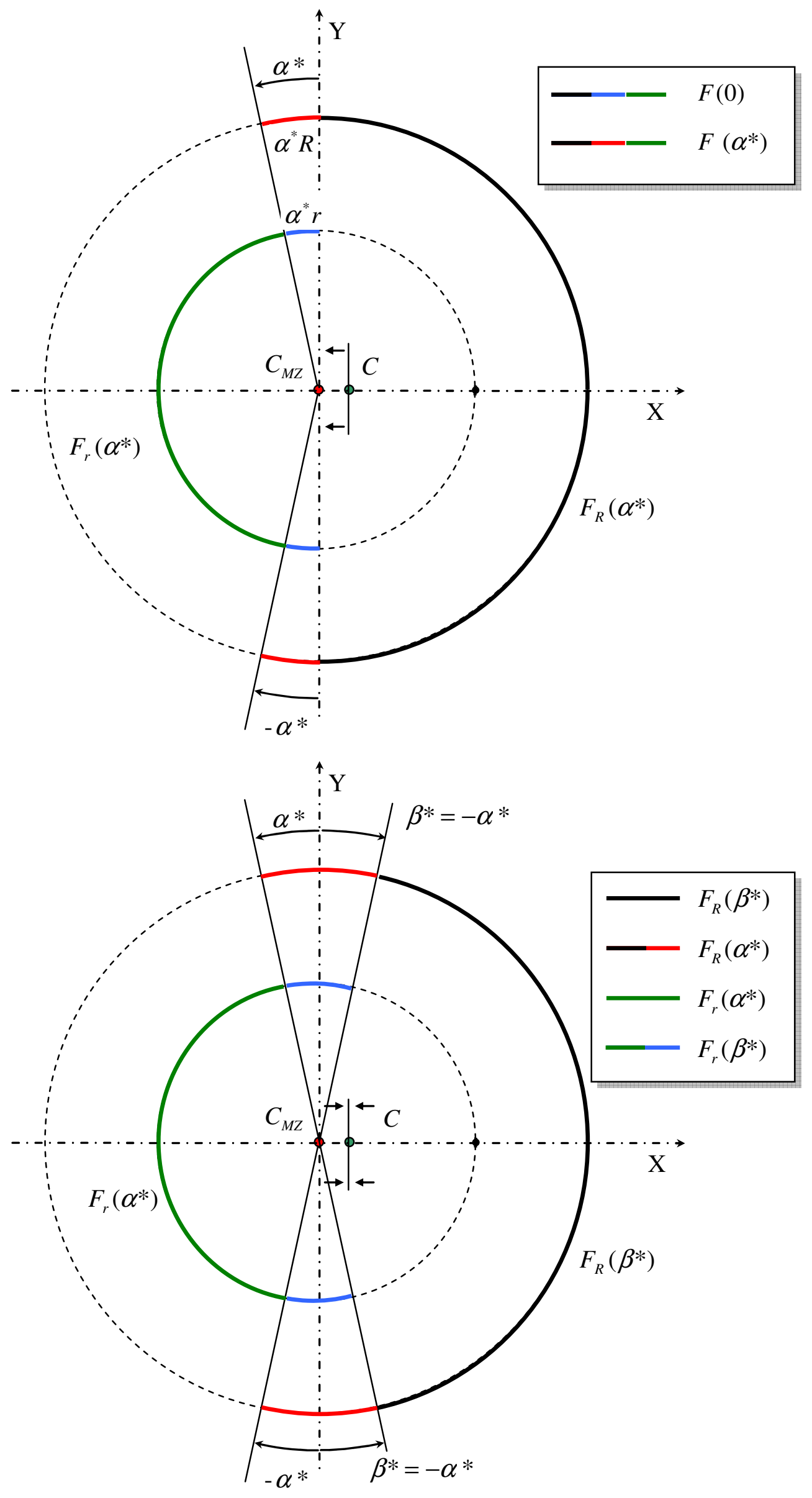


Figure 3: Graphical proof of the theorem for $-\frac{\pi}{2} \leq \alpha<\frac{\pi}{2}$. Top: composition of feature $F(\alpha)$ starting from $F(0)$. Bottom: symmetry condition about the $\mathrm{Y}$ axis between $F(\alpha *)$ and

$$
F\left(\beta^{*}\right), \beta^{*}=-\alpha^{*}
$$

About here

Reducing the asymmetry of $F(\alpha)$ about the $\mathrm{Y}$ axis, the centroid $C$ moves towards the minimum zone center $C_{M Z}$ (i.e. to the left according to Figure 3).

Theorem 1. The two concentric-opposite semicircular arcs of $F(0)$ maximize the distance $\frac{R-r}{\pi}$ given by Lemma 1 between the centroid and the origin $(0,0) \square$

- Let us suppose that a better upper bound exists, by reducing the asymmetry of the feature with $\alpha^{*} \neq 0$ and let us show that the new centroid abscissa $C^{*}$ is less than $\frac{R-r}{\pi}$ from $(0,0)$ thus contradicting the fact that reducing the asymmetry of the feature, the distance between the centroid and the minimum zone center increases.

In fact, as $\alpha^{*} \neq 0$ and $-\frac{\pi}{2} \leq \alpha<\frac{\pi}{2}$ it results:

$$
\begin{aligned}
C^{*}= & \frac{\int_{\frac{\pi}{2}-\alpha}^{\frac{\pi}{2}+\alpha} R \cdot \cos \theta \cdot d \theta+\int_{\frac{\pi}{2}+\alpha}^{\frac{3 \pi}{2}-\alpha} r \cdot \cos \theta \cdot d \theta}{\int_{0}^{2 \pi} d \theta}=\frac{\left.R \cdot \sin \theta\right|_{-\frac{\pi}{2}-\alpha} ^{\frac{\pi}{2}+\alpha}+\left.r \cdot \sin \theta\right|_{\frac{\pi}{2}+\alpha} ^{\frac{3 \pi}{2}-\alpha}}{2 \pi}=\frac{2 R \cdot \cos \alpha-2 r \cdot \cos \alpha}{2 \pi}= \\
= & \frac{(R-r) \cos \alpha}{\pi}<C
\end{aligned}
$$

In the case of $\alpha^{*}=0$, it results $C^{*}=C$.

From Theorem 1 it follows that, in the worst-case, the distance from the centroid to the origin $(0,0)$ is achieved when $r$ is low. This distance $D$ can be formulated as a function of the roundness error $E_{C}$ related to the centroid $C$. As a consequence, in the remainder only $F(0)$ is considered.

It can be noticed that if $r$ is high enough with respect to $R, C_{M Z}$ is located at $p_{I}=(0,0)$ because any given point belonging to $F(\alpha)$ is on the inner circumference (of radius $r$ ) or on the outer 
circumference (of radius $R$ ); $E_{M Z}$ is equal to $(R-r)$. Vice versa, if $r$ is low with respect to $R$, the smaller of the two concentric circles with minimal radial separation crosses the points $(r, 0)$ and $(R, 0): C_{M Z}$ is located on $p_{2=}\left(\frac{R+r}{2}, 0\right)$.

Lemma 2. The minimum zone center $C_{M Z}$ of $F(0)$ is at $(0,0)$ if and only if the ratio $\frac{r}{R}$ is a solution of the equation $2\left(\frac{r}{R}\right)^{2}-5 \frac{r}{R}+1 \leq 0$

- By the definition (2) of minimum zone center, $p_{I}=C_{M Z}$ if the following condition is verified:

$$
R_{\max }\left(p_{1}\right)-R_{\min }\left(p_{1}\right) \leq R_{\max }\left(p_{2}\right)-R_{\min }\left(p_{2}\right)
$$

Besides, if $p_{2}=C_{M Z}$ the greater of the two concentric circles with minimal radial separation crosses the point $(0, R)$. Hence in this case:

$$
E_{M Z}=\sqrt{\left(\frac{R+r}{2}\right)^{2}+R^{2}}-\left(\frac{R-r}{2}\right)
$$

and the expression (5) becomes:

$$
\begin{aligned}
& R-r \leq \sqrt{\left(\frac{R+r}{2}\right)^{2}+R^{2}}-\left(\frac{R-r}{2}\right) \\
& 3\left(1-\frac{r}{R}\right) \leq \sqrt{5+2 \frac{r}{R}+\left(\frac{r}{R}\right)^{2}} \\
& 2\left(\frac{r}{R}\right)^{2}-5 \frac{r}{R}+1 \leq 0
\end{aligned}
$$

Corollary. The interval of feasible solutions of expression (7) is $\frac{5-\sqrt{17}}{4} \leq \frac{r}{R}<1$ because the contiguous interval $1 \leq \frac{r}{R} \leq \frac{5+\sqrt{17}}{4}$ includes unfeasible solutions for $F(\alpha)$.

Theorem 2. $D=\max _{F(0)}\left|C-C_{M Z}\right| \leq \pi^{-1} E_{C} \square$

- From Lemma 1 the distance $D$ between centroid and minimum zone center is maximized when $r$ is minimized.

From Theorem 2 and its Corollary, if $C_{M Z}=(0,0)$ it results: 


$$
D=\max _{F(0)}\left|C-C_{M Z}\right|=\max _{r \geq\left(\frac{5-\sqrt{17}}{4}\right) R} \frac{R-r}{\pi} \leq \frac{\sqrt{17}-1}{4 \pi} R
$$

Equation (6) can be used to evaluate $E_{C}$. Let $R C_{C}$ and $R I_{C}$ be the radii of, respectively, the minimum circumscribed and the maximum inscribed concentric circles centered at the centroid. By expression (6) it results:

$$
\begin{aligned}
& R C_{C}=\sqrt{\left(\frac{R+r}{2}\right)^{2}+R^{2}} \\
& R I_{C}=\frac{R-r}{2}
\end{aligned}
$$

Therefore:

$$
\begin{aligned}
E_{C} & =R C_{C}-R I_{C}= \\
& =R\left(\sqrt{\left(\frac{1+\frac{r}{R}}{2}\right)^{2}+1}-\left(\frac{1-\frac{r}{R}}{2}\right)=\right. \\
& =\frac{R}{2}\left(\sqrt{5+2 \frac{r}{R}+\left(\frac{r}{R}\right)^{2}}-1+\frac{r}{R}\right) \geq \\
& \geq \frac{R}{2}\left(\sqrt{5+\frac{5-\sqrt{17}}{2}+\left(\frac{5-\sqrt{17}}{4}\right)^{2}}-\frac{\sqrt{17}-1}{4}\right)= \\
& =\frac{R}{2}\left(\sqrt{\frac{81-9 \sqrt{17}}{8}}-\frac{\sqrt{17}-1}{4}\right)= \\
& =\frac{\sqrt{17}-1}{4} R
\end{aligned}
$$

From equations (8) and (11):

$$
\frac{D}{E_{C}} \leq \frac{k}{\pi k}, \text { where } k=\frac{\sqrt{17}-1}{4}
$$

Finally, if $C_{M Z} \neq(0,0)$ it is located on $p_{2=}\left(\frac{R+r}{2}, 0\right)$, which is closer to $C$ than $p_{1=}(0,0)$. In fact:

$$
\left|p_{2}-C\right|=\frac{R+r}{2}-\frac{R-r}{\pi} \leq \frac{R-r}{\pi}=\left|p_{1}-C\right| \Leftrightarrow \frac{r}{R} \leq \frac{4-\pi}{4+\pi}=0.12
$$

Expression (13) is verified because for the Corollary in this case $\frac{r}{R}<\frac{5-\sqrt{17}}{4}=0.219$ 


\section{Application}

To show a possible application of the method and to provide some orders of magnitude of the estimation of the minimum zone center $C_{M Z}$ found by different authors, its distance $\left|C-C_{M Z}\right|$ from the centroid $C$ on datasets from the literature has been compared in Table 1 with the upper bound $\pi$ ${ }^{1} E_{C_{n}}$ predicted by the theory proven in the previous section. A similar comparison is reported in Table 2 with datasets obtained by NPL Chebyshev best fit circle certified software [24], where the exact minimum zone center is known by construction. By this analysis on concrete data, the position of the minimum zone center within the (theoretical) search area $S$ is estimated. 
Table 1: The proposed upper bound $\left(\pi^{-1} E_{C_{n}}\right.$ ) compared with the distance between centroid $C$ and minimum zone center $C_{M Z}$ (estimated) on roundness profiles taken from the literature.

\begin{tabular}{|c|c|c|c|c|c|c|c|c|c|c|c|c|c|}
\hline \multirow{2}{*}{$\begin{array}{l}\# \\
\# \\
\ddot{\Xi} \\
\Xi\end{array}$} & \multirow{2}{*}{ 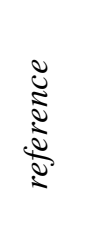 } & \multicolumn{3}{|c|}{ dataset } & \multirow[t]{2}{*}{ algorithm } & \multicolumn{3}{|c|}{ centroid $C$} & \multicolumn{2}{|c|}{$\begin{array}{l}\text { minimum zone center } \\
\qquad C_{M Z}\end{array}$} & \multirow[t]{2}{*}{$\left|C-C_{M Z}\right|$} & \multirow[t]{2}{*}{$\pi^{-1} E_{C_{n}}$} & \multirow[t]{2}{*}{$\begin{array}{c}\text { difference } \\
\text { (14) }\end{array}$} \\
\hline & & author & $n$ & notes & & $x_{0}$ & $y_{0}$ & $E_{C_{n}}$ & $x_{M Z}$ & $y_{M Z}$ & & & \\
\hline 1. & {$[3]$} & [3] & 1800 & $\begin{array}{l}\text { Drilling/aluminum } \\
\text { alloy/CMM data }\end{array}$ & GA & -0.0002 & $0.0 \mathrm{n} 022$ & 0.0301 & -0.0026 & 0.0044 & 0.003256 & 0.009581 & +0.006325 \\
\hline 2. & {$[3]$} & {$[3]$} & 1800 & $\begin{array}{l}\text { Milling/steel/ } \\
\text { CMM data }\end{array}$ & GA & 0.0042 & 0.0008 & 0.0242 & 0.0071 & -0.0018 & 0.003895 & 0.007703 & +0.003808 \\
\hline 3. & [3] & {$[3]$} & 1800 & $\begin{array}{l}\text { Turning/steel/ } \\
\text { CMM data }\end{array}$ & GA & 0.0007 & -0.0048 & 0.0270 & 0.0010 & -0.0049 & 0.000308 & 0.008594 & +0.008287 \\
\hline 4. & {$[3]$} & {$[3]$} & 1800 & $\begin{array}{l}\text { Drilling/marble } \\
\text { CMM data }\end{array}$ & GA & 0.0040 & -0.0199 & 0.0688 & 0.0031 & -0.0181 & 0.002012 & 0.021263 & +0.019251 \\
\hline 5. & [3] & {$[3]$} & 1800 & $\begin{array}{l}\text { Turning/steel } \\
\text { CMM data }\end{array}$ & GA & 0.0037 & -0.0199 & 0.0709 & -0.0060 & -0.0137 & 0.009801 & 0.022568 & +0.012768 \\
\hline 6. & {$[3]$} & [3] & 1800 & $\begin{array}{l}\text { Turning/aluminum } \\
\text { alloy CMM data }\end{array}$ & GA & -0.0115 & -0.0112 & 0.0935 & -0.0178 & -0.0068 & 0.007684 & 0.029762 & +0.022078 \\
\hline 7. & {$[3]$} & {$[3]$} & 1800 & $\begin{array}{l}\text { Turning/steel } \\
\text { CMM data }\end{array}$ & GA & -0.0021 & 0.0010 & 0.0423 & -0.0071 & 0.0014 & 0.005016 & 0.013465 & +0.008449 \\
\hline 8. & [14] & [17] & 39 & Artificial data & GA & 0.0356 & -0.0536 & 0.0092 & 0.0356 & -0.0529 & 0.0007 & 0.002928451 & +0.002228451 \\
\hline 9. & [14] & {$[25]$} & 100 & Artificial data & GA & 0.02908 & -0.00634 & 0.9868 & 0.00536 & 0.00788 & 0.027655864 & 0.314108196 & +0.286452332 \\
\hline 10. & [14] & [10] & 24 & CMM data & GA & 82.989744 & 97.009116 & 0.039058 & 82.990941 & 97.008387 & 0.001401517 & 0.012432548 & +0.01103103 \\
\hline
\end{tabular}




\begin{tabular}{|c|c|c|c|c|c|c|c|c|c|c|c|c|c|}
\hline 11. & {$[26]$} & {$[26]$} & 8 & Artificial data & $\begin{array}{c}\text { Computational } \\
\text { Geometric }\end{array}$ & 29.997 & 40.0606 & 0.30177 & 29.96886 & 40.10828 & 0.055342 & 0.096056 & +0.040715 \\
\hline 12. & {$[10]$} & [19] & 24 & CMM data & $\begin{array}{l}\text { Steepest } \\
\text { Descend }\end{array}$ & 82.989744 & 97.009116 & 0.039058 & 82.99094 & 97.00839 & 0.001404 & 0.012433 & +0.011029 \\
\hline 13. & {$[10]$} & {$[10]$} & 25 & CMM data & $\begin{array}{l}\text { Steepest } \\
\text { Descend }\end{array}$ & 40.0007 & 50.0015 & 0.0293 & 40.00074 & 50.00153 & 0.0000492 & 0.009326 & +0.009277 \\
\hline 14. & {$[20]$} & [20] & 80 & $\begin{array}{c}\mathrm{CMM} / \text { Bearing } \\
\text { ring }\end{array}$ & $\begin{array}{c}\text { Polar } \\
\text { Coordinate } \\
\text { Transform }\end{array}$ & 0 & 0 & 0.029 & 0.001599 & -0.00165 & 0.0023 & 0.009231 & +0.006931 \\
\hline 15. & {$[20]$} & [20] & 80 & $\begin{array}{c}\mathrm{CMM} / \text { Bearing } \\
\text { ring }\end{array}$ & $\begin{array}{c}\text { PTC } \\
8 \times 80 \text { mesh }\end{array}$ & 0 & 0 & 0.029 & 0.001422 & -0.0023 & 0.002704 & 0.009231 & +0.006527 \\
\hline 16. & {$[20]$} & {$[20]$} & 80 & $\begin{array}{c}\mathrm{CMM} / \text { Bearing } \\
\text { ring }\end{array}$ & $\begin{array}{c}\text { PTC } \\
10 \times 120 \text { mesh }\end{array}$ & 0 & 0 & 0.029 & 0.001592 & -0.00166 & 0.0023 & 0.009231 & +0.006931 \\
\hline 17. & {$[20]$} & {$[20]$} & 80 & $\begin{array}{c}\mathrm{CMM} / \text { Bearing } \\
\text { ring }\end{array}$ & $\begin{array}{c}\text { PTC } \\
8 \times 100 \text { mesh }\end{array}$ & 0 & 0 & 0.029 & 0.001372 & -0.00233 & 0.002704 & 0.009231 & +0.006527 \\
\hline 18. & {$[20]$} & [20] & 80 & $\begin{array}{c}\mathrm{CMM} / \text { Bearing } \\
\text { ring }\end{array}$ & $\begin{array}{c}\text { PTC } \\
20 \times 180 \text { mesh }\end{array}$ & 0 & 0 & 0.029 & 0.001573 & -0.00168 & 0.002301 & 0.009231 & +0.006930 \\
\hline 19. & {$[27]$} & {$[27]$} & 100 & Artificial data & $\begin{array}{l}\text { Approximation } \\
\text { Algorithm }\end{array}$ & 0 & 0 & 0.04 & $\begin{array}{c}- \\
0.00000002\end{array}$ & $\begin{array}{c}- \\
0.00000001\end{array}$ & 0.00000002 & 0.012732 & +0.012732 \\
\hline 20. & {$[27]$} & {$[27]$} & 1000 & Artificial data & $\begin{array}{c}\text { Approximation } \\
\text { Algorithm }\end{array}$ & 5 & -4 & 0.02 & 5.00000016 & $\begin{array}{c}- \\
4.00000054\end{array}$ & 0.0000006 & 0.006366 & +0.006366 \\
\hline
\end{tabular}


Table 2: The proposed upper bound $\left(\pi^{-1} E_{C_{n}}\right)$ compared with the distance between centroid $C$ and minimum zone center $C_{M Z}$ (known) on roundness profiles obtained by certified software [24] with increasing dataset size $n$, predefined minimum zone error $E_{M Z}$ and center $C_{M Z}=\left(x_{M Z}, y_{M Z}\right) \equiv(0,0)$.

\begin{tabular}{|c|c|c|c|c|c|c|c|c|}
\hline \multirow{2}{*}{ 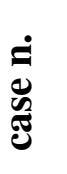 } & \multirow[t]{2}{*}{$n$} & \multirow[t]{2}{*}{$\boldsymbol{E}_{M Z}$} & \multicolumn{3}{|c|}{$C$} & \multirow[t]{2}{*}{$\left|C-C_{M Z}\right|$} & \multirow[t]{2}{*}{$\boldsymbol{\pi}^{\mathbf{1}} E_{C_{n}}$} & \multirow{2}{*}{$\begin{array}{c}\text { difference } \\
\text { (14) }\end{array}$} \\
\hline & & & $x_{0}$ & $y_{0}$ & $E_{C_{n}}$ & & & \\
\hline 1. & 8 & 0.01 & -0.0009 & -0.0002 & 0.0113 & 0.0007 & 0.0036 & +0.0029 \\
\hline 2. & 16 & 0.03 & -0.0001 & -0.0025 & 0.0333 & 0.0024 & 0.0106 & +0.0082 \\
\hline 3. & 32 & 0.06 & -0.0007 & 0.0030 & 0.0632 & 0.0031 & 0.0201 & +0.0170 \\
\hline 4. & 64 & 0.09 & -0.0030 & 0.0008 & 0.0940 & 0.0031 & 0.0299 & +0.0268 \\
\hline 5. & 128 & 0.01 & -0.0002 & -0.0001 & 0.0102 & 0.0002 & 0.0032 & +0.0030 \\
\hline 6. & 256 & 0.03 & 0.0004 & 0.0002 & 0.0308 & 0.0004 & 0.0098 & +0.0094 \\
\hline 7. & 512 & 0.06 & -0.004 & -0.0007 & 0.0612 & 0.0008 & 0.0195 & +0.0187 \\
\hline 8. & 1024 & 0.09 & 0.0010 & 0.0001 & 0.0915 & 0.0010 & 0.0291 & +0.0281 \\
\hline 9. & 2048 & 0.01 & 0.0000 & 0.0000 & 0.0101 & 0.0000 & 0.0032 & +0.0032 \\
\hline 10. & 4096 & 0.03 & 0.0001 & 0.0000 & 0.0301 & 0.0001 & 0.0096 & +0.0095 \\
\hline 11. & 8192 & 0.06 & 0.0001 & -0.0001 & 0.0602 & 0.0001 & 0.0192 & +0.0190 \\
\hline 12. & 16384 & 0.09 & -0.0002 & 0.0000 & 0.0903 & 0.0002 & 0.0287 & +0.0285 \\
\hline
\end{tabular}


As anticipated in the introduction, not all the minimum zone methods are based on the minimum zone center $C_{M Z}$ to determine the minimum zone roundness error and are able to provide its position. From our extensive search, all center-based methods found that provide the $C_{M Z}$ have been included in Table 1 . The listed $C_{M Z}$ values are not necessarily optima; they have been obtained by the respective authors by their proposed method, as specified. Table 2 includes datasets where the $C_{M Z}$ is known. The estimate is quantified by the following parameter

$$
\text { difference }=\pi^{-1} E_{C_{n}}-\left|C-C_{M Z}\right|
$$

and is always positive as an experimental evidence of the proposed theory.

It can be noticed that the proposed upper bound $\pi^{-1} E_{C}$ ranges from the double to one order of magnitude above the estimated $C$ to $C_{M Z}$ distance in most cases, regardless of the magnitude of $E_{C}$. The same can be observed for Table 2 .

It can also be observed for both tables that the difference increases both with the dataset size and with the minimum zone error.

The first seven roundness profiles in Table 1 have been taken by the authors from real samples on a variety of materials and manufacturing processes, are visually shown in [3] and include different shapes, like flattened circle, three lobed part, elliptic form, circle with random deviations etc.

Form deviations are not considered by the proposed theory, which provides a general result, e.g. it is not signature specific, however it is speculated that the upper bound provides a larger overestimate (difference by expression (14)) with high form errors (high $E_{C_{n}}$ ) and low profile deviations $\left(C_{M Z}\right.$ close to $\left.C\right)$.

\section{Profile sampling}

In Figure 4, the same comparison of previous section is repeated on roundness profiles at increasing sampling size $n$.

36 datasets have been generated with certified software [24] and minimum zone error 0.01, 0.03 and $0.06 \mathrm{~mm}$ for 12 values of the sampling size $n$ in the range 8 to 16,384 . The deviation type with generated profiles is random, i.e. the error is uniformly distributed around the $360^{\circ}$. 


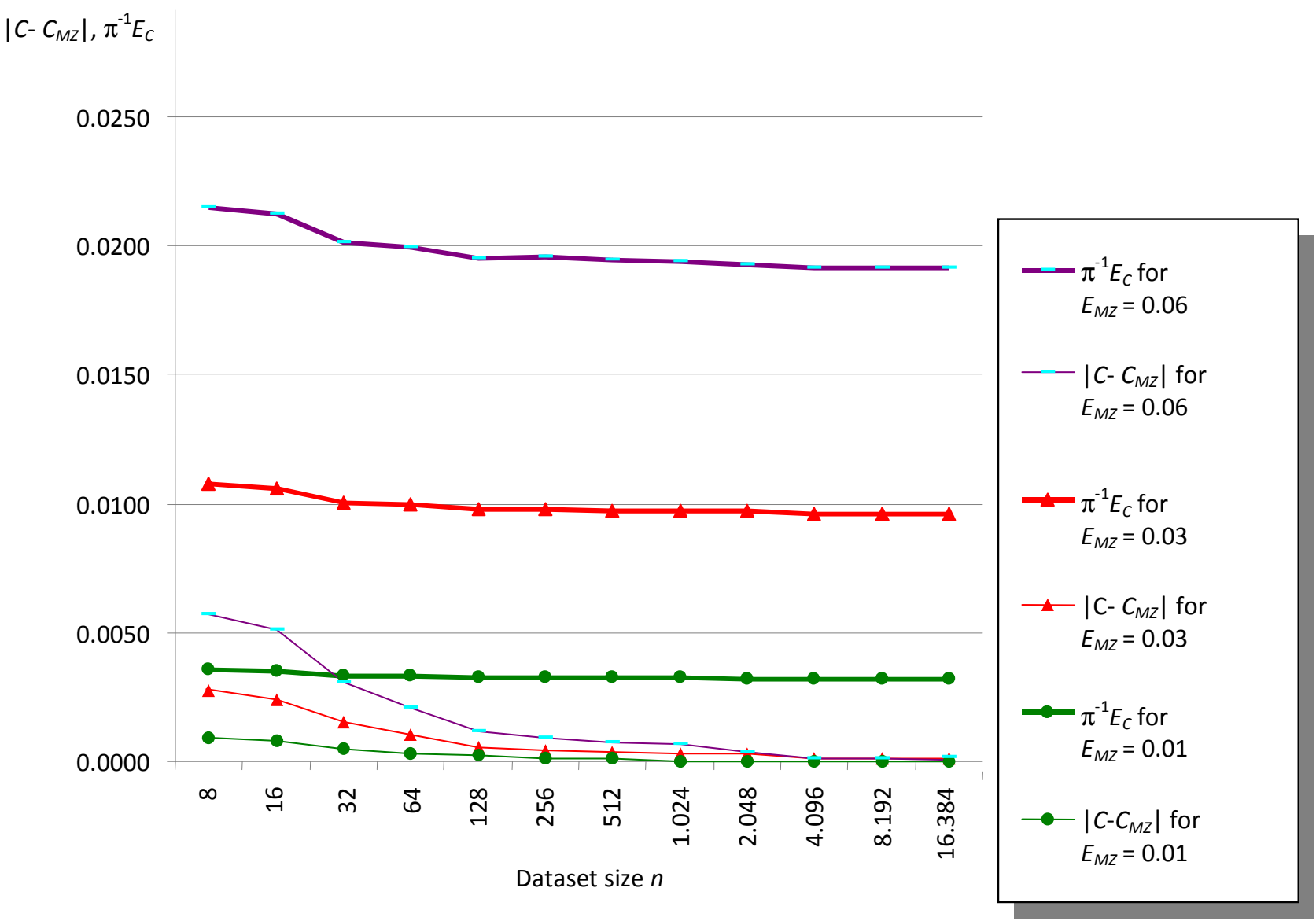

Figure 4: The proposed upper bound $\pi^{-1} E_{C}$ compared to the distance between the centroid and the minimum zone center fixed in $(0,0)$ of roundness profiles generated by certified software [24].

About here

As in Table 2, the minimum zone center in Figure 4 is known; it has been fixed at the origin of coordinates $(0,0)$ in the profile generation; its distance from the centroid and the respective upper bound $\pi^{-1} E_{C}$ proposed in this paper have been compared.

The developed theory is valid for continuous profiles and consequently at increasing sampling size, when dealing with discrete data; however, it is satisfied already for $n$ as low as 8 . It should also be considered that in applications requiring better accuracy on the MZE estimation, $n$ should be higher, up to thousands datapoints, which are available with optical scanning techniques. The trend on exact data is clearly monotonously decreasing as expected.

\section{Discussion and conclusions}

The worst-case described in Figure 2 has been built to achieve a closed form evaluation of the centroid to minimum zone center distance. By knowing the centroid of the roundness profile, the minimum zone center position can be estimated with a tolerance whose upper bound has been given in closed form by $\pi^{-1} E_{C}$. 
The worst-case also expresses concrete manufacturing cases, such as casting or forming where two dies or molds have a different radius.

The roundness error $E_{C_{n}}$ related to the centroid of a dataset with $n$ equiangular data can be evaluated in linear function of the sampling data; increasing the number of sampled points, the estimate accuracy increases.

This theory has extensive applications.

- The part center can be located with a given tolerance in manufacturing tasks, such as handling (peg-hole) or machining (centering).

- Based on the worst-case approach, these conclusions can be extended to any sampling profile also defined as blind or not manufacturing signature-specific.

- By a transformation from polar to Cartesian coordinates, the proposed upper bound can be directly applied to the estimation of straightness.

- Minimum zone based algorithms and algorithms that approach the minimum zone method by iterative center evaluations (e.g. metaheuristics like genetic algorithms or particle swarm optimization and others cited above) can benefit of the lower search space size to a neighbor of the centroid $C$.

For these latter center-based minimum zone methods, current result represents a new opportunity for experimental research.

The theoretical upper bound proposed is not signature specific, however it is speculated that a larger overestimate is obtained with high errors (high $\left.E_{C_{n}}\right)$ and low profile deviations $\left(C_{M Z}\right.$ close to $C$ ). The correlation between the proposed upper bound and form deviations can be investigated in future research.

Future work includes the extension of the proposed theory to other (symmetrical) form errors, such as sphericity and planarity.

\section{Acknowledgements}

The authors would like to thank Mr. Stefano Chiodi for the insightful observations to Theorem 1 and the anonymous referees for stimulating the discussion on practical implications.

\section{Appendix}

The two concentric-opposite arcs feature can be parameterized by: 


$$
F(x, \alpha)= \begin{cases}(x \cdot \cos \theta, 0), & \theta=0 \\ (R \cdot \cos \theta, R \cdot \sin \theta), & \theta \in\left(0, \frac{\pi}{2}+\alpha\right] \\ (r \cdot \cos \theta, r \cdot \sin \theta), & \theta \in\left(\frac{\pi}{2}+\alpha, \frac{3 \pi}{2}+\alpha\right) \\ (R \cdot \cos \theta, R \cdot \sin \theta), & \theta \in\left[\frac{3 \pi}{2}+\alpha, 0\right)\end{cases}
$$

where $-\frac{\pi}{2} \leq \alpha<\frac{\pi}{2}$ and $0 \leq x \leq R$.

Proposition. $E_{M Z}$ is un upper bound of $(R-r)$ when a control point $(x, 0)$ moves from $r$ to 0 on the $\mathrm{X}$ axis $\square$

- The (1-3) minimum zone condition is considered: the one-point taken on the inner circumference is exactly $(x, 0)$; the three-points taken on the outer circumference have center $C_{M Z}$. Therefore $E_{M Z}=R-x \geq R-r$

Besides, it can be observed that $E_{M Z}$ is included in the region delimited by $(R-x)$ and $(R-r)$ when the point $x$ moves from $r$ to $\mathrm{G}$ on the $\mathrm{X}$ axis, where $\mathrm{G}$ is the circumcenter of vertexes $\{(r \cdot \operatorname{sen} \alpha, r \cdot \cos \alpha),(r \cdot \operatorname{sen} \alpha,-r \cdot \cos \alpha),(R, 0)\}$ (not proven here). Finally, $E_{M Z}$ is equal to $(R-r)$ for $G \leq x \leq R$. 


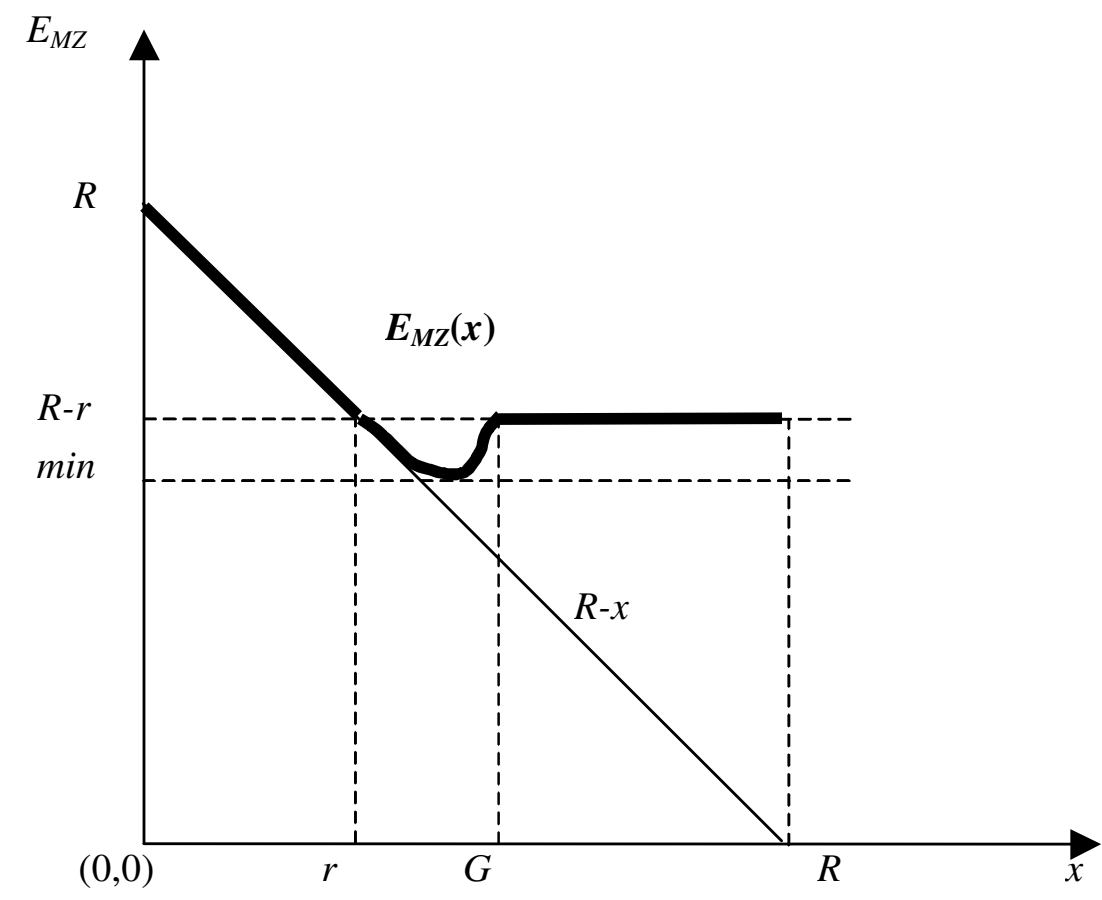

Figure A: Qualitative effect of the variation of the control point $x \in[0, R]$ on the minimum zone error $E_{M Z} \cdot$

About here

\section{References}

[1] G. Moroni, S. Petro, Geometric tolerance evaluation: A discussion on minimum zone fitting algorithms, Precision Engineering, 32 (3) July (2008), pp. 232-237, ISSN 0141-6359, doi:10.1016/j.precisioneng.2007.08.007.

[2] International Organization for Standardization, Geneva, Switzerland, ISO 1101, Geometrical Product Specifications (GPS)-tolerances of form, orientation, location and run out, 2nd ed.; December (2004).

[3] A. Rossi, M. Antonetti, M. Barloscio. M. Lanzetta, Fast genetic algorithm for roundness evaluation by the minimum zone tolerance (MZT) method, Measurement, 44 (7) August (2011), ISSN 0263-2241, doi:10.1016/j.measurement.2011.03.031, pp. 1243-1252.

[4] A. Rossi, M. Lanzetta, Optimal blind sampling strategy for minimum zone roundness evaluation by metaheuristics, Precision Engineering, 37 (2) (2013), pp. 241-247 doi:10.1016/j.precisioneng.2012.09.001.

[5] U. Roy, X. Zhang, Establishment of a pair of concentric circles with the minimal radial separation for assessing roundness error, Computer Aided Design, 24(2) (1992), pp. 161-168.

[6] X. Li, H. Liu, W. Li, Development and application of $\alpha$-hull and Voronoi diagrams in the assessment of roundness error, Measurement Science and Technology, 22 (2011), 045105 doi:10.1088/0957-0233/22/4/045105.

[7] P.B. Dhanish, M.S. Shunmugam, An algorithm for form error evaluation - using the theory of discrete and linear chebyshev approximation, Computer Methods in Applied Mechanics and Engineering, 92 (1991), pp. 309-324. 
[8] T. Weber, S. Motavalli, B. Fallahi, S.H. Cheraghi, A unified approach to form error evaluation, Journal of the International Societies for Precision Engineering and Nanotechnology, 26 (2002), pp. 269-278.

[9] T.S.R. Murthy, S.Z. Abdin, Minimum zone evaluation of surfaces, International Journal of Machine Tool Design Research, 20 (1980), pp. 123-136.

[10] L.M. Zhu, H. Ding, Y.L. Xiong, A steepest descent algorithm for circularity evaluation, Computer Aided Design, 35 (3) (2003), pp. 255-265.

[11] Y. Kovvur, H. Ramaswami, B.R. Anand, S. Anand, Minimum-zone form tolerance evaluation using particle swarm optimisation, International Journal of Intelligent Systems Technologies and Applications, 4 (1/2) (2008).

[12] J. Mao, Y. Cao, J. Yang, Implementation uncertainty evaluation of cylindricity errors based on geometrical product specification (GPS), Measurement, 42 (5) June (2009), pp. 742-747.

[13] C.M. Shakarji, A. Clement, Reference Algorithms for Chebyshev and One-Sided Data Fitting for Coordinate Metrology, CIRP Annals - Manufacturing Technology, 53 (1) (2004), pp. 439442.

[14] X. Wen, Q. Xia, Y. Zhao, An effective genetic algorithm for circularity error unified evaluation, International Journal of Machine Tools \& Manufacture, 46 (2006), pp. 17701777.

[15] C. Cui, B. Li, F. Huang, R. Zhang, Genetic algorithm-based form error evaluation, Measurement Science and Technology, 18 (2007), pp. 1818, doi:10.1088/09570233/18/7/004.

[16] M. Wang, S.H. Cheraghi, A.S.M. Masud, Circularity error evaluation: theory and algorithm, Precision Engineering, 23 (3) (1999), pp. 164-76.

[17] W.-Y. Jywe, G.-H, Liu, C.-K. Chen, The min-max problem for evaluating the form error of a circle, Measurement, 26 (1999), pp. 273-282.

[18] E.S. Gadelmawla, Simple and efficient algorithm for roundness evaluation from the coordinate measurement data, Measurement, 43 (2010), pp. 223-235.

[19] G.L. Samuel, M.S. Shunmugam, Evaluation of circularity from coordinate and form data using computational geometric techniques, Precision Engineering, 24 (2000), pp. 251-263.

[20] X. Lei, C. Zhang, Y. Xue, J. Li., Roundness Error Evaluation Algorithm Based on Polar Coordinate Transform, Measurement, 44 (2) February (2011), pp. 345-350.

[21] A. Rossi, A form of deviation-based method for coordinate measuring machine sampling optimization in an assessment of roundness, Proceedings of the Institution of Mechanical Engineers, Part B: Journal of Engineering Manufacture, 215 (2001), pp. 1505-1518.

[22] R. Sharma, K. Rajagopal, S. Anand, A Genetic Algorithm Based Approach for Robust Evaluation of Form Tolerances, Journal of Manufacturing Systems, 19 (1) (2000), pp. 46-57.

[23] L. Yan, B. Yan, L. Cai, G. Hu, M. Wang, Research on roundness error evaluation of shaft parts based on genetic algorithms with transfer-operator, 9th International Conference on Electronic Measurement \& Instruments, ICEMI '09, (2009), pp. 2-362 - 2-366. doi:10.1109/ICEMI.2009.5274566.

[24] National Physical Laboratory (UK), Data Generator for Chebyshev Best-Fit Circle, http://www.npl.co.uk/mathematics-scientific-computing/software-support-for-metrology/, last acc. March 2013.

[25] J. Huang, A new strategy for circularity problems, Precision Engineering, 25 (4) (2001), pp. 301-308.

[26] P.B. Dhanish, A simple algorithm for evaluation of minimum zone circularity error from coordinate data. International Journal of Machine Tools and Manufacture, 42 (14) (2002), pp. 1589-1594. 
[27] G. Goch, K. Lübke, Tschebyscheff approximation for the calculation of maximum inscribed/minimum circumscribed geometry elements and form deviations, CIRP Annals Manufacturing Technology, 57 (2008), pp. 517-520. 